\title{
A MAXIMUM MODULUS PROPERTY OF MAXIMAL SUBALGEBRAS
}

\author{
PAUL CIVIN ${ }^{1}$
}

In a recent paper [6] Wermer considered the algebra $C$ of all continuous complex valued functions on $\gamma$, a simple closed analytic curve bounding a region $\Gamma$, with $\Gamma \cup_{\gamma}$ compact, on a Riemann surface $F$. He considered the subalgebra $A$ of all functions in $C$ which could be extended into $\Gamma$ to be analytic on $\Gamma$ and continuous on $\Gamma \cup_{\gamma}$. Wermer showed that $A$ was a maximal closed subalgebra of $C$ which separated the points of $\gamma$, and that the space of maximal ideals of $A$ was homeomorphic to $\Gamma \cup_{\gamma}$.

In [2] Civin and Yood considered a class of subalgebras of complex commutative regular Banach algebras which become maximal closed subalgebras in the event the original algebra was the collection of continuous functions on a compact Hausdorff space. The object of this note is to demonstrate that such subalgebras possess a maximum modulus property possessed by $A$. To state the result obtained we recall certain definitions. The terms not herein defined may be found in $[5]$.

Let $B$ be a complex commutative regular Banach algebra with identity $e$ and space of maximal ideals $\mathfrak{M}(B)$. Let $\pi: x \rightarrow x(M)$ be the Gelfand representation of $B$ as a subalgebra of $C(\mathfrak{M}(B))$, the continuous function on $\mathfrak{M}(B)$. We also denote $\pi x$ by $\hat{x}$ and $\pi Q$ by $\hat{Q}$ for any subset $Q$ of $B$. A subalgebra $N$ of $B$ is called determining [2] if $\pi N$ is dense in $\pi B$, otherwise $N$ is called nondetermining. A subalgebra of $B$ is called a maximal nondetermining subalgebra if every larger subalgebra of $B$ is deternining. A subset $S$ of $B$ is called a separating family on $\mathfrak{M}(B)$ if for each $M_{1}, M_{2}$ in $\mathfrak{M}(B), M_{1} \neq M_{2}$, there exists an $x \in S$ such that $x\left(M_{1}\right) \neq x\left(M_{2}\right)$. If $P$ is an algebra of continuous complex valued functions vanishing at infinity on the locally compact space $X$, the smallest closed set (if it exists) on which each $|f|$ with $f \in P$ assumes its maximum is called the Silov boundary of $X$ with respect to $P$.

THEOREM 1. Let $B$ be a complex commutative regular Banach algebra with identity $e$, and let $N$ be a maximal nondetermining subalgebra of

Presented to the Society, April 20, 1957; received by the editors October 16, 1958.

1 This work was supported in part by the National Science Foundation research gra t NSF-G 2573. 
$B$ which is not a maximal ideal. If $N$ is a separating family on $\mathfrak{M}(B)$, then $\mathfrak{M}(B)$ may be topologically embedded if $\mathfrak{M}(N)$ and as so embedded $\mathfrak{M}(B)$ is the Silov boundary of $\mathfrak{M}(N)$ with respect to $N$.

While the present note was in the process of publication, two proofs of Theorem 1 appeared for the special case when $B=C(X)$ for a compact Hausdorff space $X$, one by H. S. Bear [1] and the other by K. Hoffman and I. M. Singer [4].

Before proceeding to the proof of the theorem, we require one lemma, which was noted by Helson and Quigley [3] for the case $B=C(X)$.

Lemma 2. Let $N$ be a maximal nondetermining subalgebra of the complex commutative regular Banach algebra $B$, and let e be the identity of $B$. Then either $e \in N$ or $N$ is a maximal ideal of $B$.

Suppose $e \notin N$. Let $D=\{a+\lambda e: a \in N$ and $\lambda$ complex $\}$. As $e$ is the unit for $B, D$ is a subalgebra of $B$ which properly contains $N$, hence $\hat{D}$ is dense in $\hat{B}$. Let $x \in B$ and $a \in N$. There exists $a_{n} \in N$ and $\lambda_{n}$ complex, $n=1,2, \cdots$, such that $\pi\left(a_{n}+\lambda_{n} e\right) \rightarrow \pi x$ as in $n \rightarrow \infty$. Therefore $\left(a_{n}+\lambda_{n} e\right) a \in N$ and $\pi\left\{\left(a_{n}+\lambda_{n} e\right) a\right\} \rightarrow \pi(x a)$. By Lemma 1 of [2], $\hat{N}$ is closed in $\hat{B}$, so $\pi(x a) \in \hat{N}$. There thus exists $u \in N$ such that $x a-u$ is in the radical of $B$. As noted in [2], $N$ contains the radical of $B$. Thus $x a \in N$ and $N$ is an ideal of $B$. That $N$ is a maximal ideal is an immediate consequence of $N$ being maximal nondetermining.

We return to the proof of Theorem 1 . Each nonzero multiplicative linear functional on $B$ is automatically one on $N$, and distinct multiplicative linear functionals on $B$ have distinct restrictions to $N$ since $N$ is a separating family on $\mathfrak{M}(B)$. There is thus a one-to-one correspondence between $\mathfrak{M}(B)$ and a subset of $\mathfrak{M}(N)$. The mapping is clearly continuous from $\mathfrak{M}(B)$ to $\mathfrak{M}(N)$ in the Gelfand topologies. As $\mathfrak{M}(B)$ is a compact Hausdorff space, the mapping is a homeomorphism. We henceforth suppose $\mathfrak{M}(B)$ is a subset of $\mathfrak{M}(N)$.

Since $N$ is a subalgebra of $B, \lim \left\|a^{n}\right\|^{1 / n}$ is independent of whether the $N$ or $B$ norm is used. Thus sup $|a(M)|$ is the same whether calculated over $\mathfrak{M}(B)$ or $\mathfrak{M}(N)$. To see that $\mathfrak{M}(B)$ is the Silov boundary of $\mathfrak{M}(N)$ with respect to $N$, it is sufficient to see that there is no proper closed subset of $\mathfrak{M}(B)$ on which each $|a(M)|, a \in N$, attains its maximum. Suppose otherwise and let $\Omega$ be a proper closed subset of $\mathfrak{M}(B)$ of the required type.

Let $M_{0} \in \mathfrak{M}(B), M_{0} \notin \Re$. If $\mathfrak{R}$ is any closed set in $\mathfrak{M}(B)$ such that $\mathfrak{R} \Omega$ and $M_{0} \notin \mathfrak{R}$, let $\mathfrak{B}$ be an open set in $\mathfrak{M}(B)$ with $M_{0} \in \mathfrak{B}$ and $\overline{\mathfrak{B}} \cap \mathfrak{R}=\overline{\mathfrak{B}}$, the closure being in $\mathfrak{M}(B)$. Let $W=W(\mathfrak{R})$ be the kernel of $\mathfrak{R}$, i.e. $W=\cap M, M \in \mathfrak{R}$. Let $R$ be the radical of $B$. Since $B$ is a regular 
Banach algebra, $W$ contains elements not in $R$. Consider the algebra $S=N+W$. The elements of $S$ are of the form $a+u, a \in N, u \in W$, since $W$ is an ideal of $B$. For $u \in W, u \notin R$, the maximum modulus of $u(M)$ is not attained on $\Omega$, so $u \notin N$, and thus $S$ contains $N$ properly. As $N$ was maximal nondetermining, $\hat{S}$ is dense in $\hat{B}$.

Let $b \in B$. There exists $a_{n} \in N, u_{n} \in W, n=1,2, \cdots$, so that if $r_{n}=a_{n}+u_{n}$, then $\hat{p}_{n} \rightarrow \hat{b}$. For $M \in \mathcal{R}, \quad\left|a_{n}(M)-a_{m}(M)\right|=\mid r_{n}(M)$ $-r_{m}(M) \mid$. Thus

$$
\sup _{M \in \mathbb{R}}\left|a_{n}(M)-a_{m}(M)\right| \leqq \sup _{M \in \mathbb{R}(B)}\left|r_{n}(M)-r_{m}(M)\right| .
$$

By the maximum modulus property of $N$ relative to $\Re \subset \mathbb{R}$,

$$
\sup _{M \in \mathfrak{M}(B)}\left|a_{n}(M)-a_{m}(M)\right| \leqq \sup _{M \in \mathfrak{M}(B)}\left|r_{n}(M)-r_{m}(M)\right| \text {. }
$$

Since $\hat{N}$ is closed [2], there exists $a_{0} \in N$ such that $\hat{a}_{n} \rightarrow \hat{a}_{0}$. There is then an element $w_{0} \in W$ such that $\hat{a}_{n} \rightarrow \hat{w}_{0}$. If $b_{0}=a_{0}+w_{0}, \hat{\boldsymbol{t}}_{n} \rightarrow \hat{b}$ and $\hat{p}_{n} \rightarrow b_{0}$, and consequently $\hat{b}-\hat{b}_{0}=0$ and $b-b_{0} \in R$. As noted in [2], $R \subset N$, so $b-b_{0} \in N$. Since $b$ was arbitrary, $B=N+W=N+W(\mathfrak{R})$.

We next show the complement of $\Re$ in $\mathfrak{M}(B)$ consists of a single point. Suppose otherwise. Let $M_{i} \in \mathfrak{M}(B), M_{i} \notin \Omega, i=1,2$, and $M_{1} \neq M_{2}$. Let $\mathbb{R}$ be a closed set in $\mathfrak{M}(B)$, such that $\left\{M_{1}\right\} \cup \Re \subset \mathbb{R}$ and $M_{2} \in \mathbb{R}$. Since $B$ is a regular Banach algebra, there is an element $b \in B$, such that $b(M)=0, M \in \Omega$, and $b\left(M_{1}\right)=1$. We may express $b$ as $b=a+u, a \in N, u \in W(\Omega)$. For $M \in \Omega, 0=b(M)=a(M)+u(M)$. Since $u(M)=0$ for $M \in \Omega, a(M)=0$ for $M \in \Re$. However, $1=b\left(M_{1}\right)=a\left(M_{1}\right)$ $+u\left(M_{1}\right)=a\left(M_{1}\right)$ since $M_{1} \in \mathbb{R}$. This contradicts the supposition that for $a \in N$,

$$
\sup _{M \in \Re}|a(M)|=\sup _{M \in \mathfrak{M}(B)}|a(M)| .
$$

Thus $\mathfrak{M}(B)=\mathfrak{R} \cup\left\{M_{0}\right\}$, and since $\Re$ was closed in $\mathfrak{M}(B), M_{0}$ is an isolated point of $\mathfrak{M}(B)$.

Let $W=W(\Re)=\{f \in B \mid \hat{f}(\Re) \equiv 0\}$. Consider any element $b+W$ of $B / W$. Since $b=a+u$, with $a \in N, u \in W$, there is an element $a$ of $N$ in the coset $b+W$. Now $R \subset W$, so all elements of the coset $a+R$ of $N / R$ are in the coset $b+W$. Moreover if $a_{i} \in b+W$, and $a_{i} \in N$, $i=1,2$, then $a_{1}-a_{2} \in W$ so by the maximum modulus property that $\Omega$ is alleged to have $\hat{a}_{1}-\hat{a}_{2}=0$ and $a_{1}-a_{2} \in R$. There is thus a oneto-one correspondence between the cosets $b+W$ and $a+R$. The correspondence gives an isomorphism of $B / W$ and $N / R$.

Let $N_{1}=\left\{a \in N: a\left(M_{0}\right)=0\right\}$. Then $N_{1}$ is a maximal ideal of $N$ which contains $R$ and thus $N_{1} / R$ is a maximal ideal of $N / R$. The iso- 
morphism obtained above implies the existence of a maximal ideal $M_{1}$ in $B, M_{1} \supset W$ and with $M_{1} / W$ isomorphic to $N_{1} / R$. Since $M_{1} \supset W$, $M_{1} \neq M_{0}$.

Let $a \in N_{1}$, so $a\left(M_{0}\right)=0$. Then $a\left(M_{1}\right)=0$ because of the inclusion of the coset $a+R$ in the coset $a+W$. Similarly, if $a \in N \cap M_{1}$, then $a \in M_{0}$. Lemma 2 implies that for arbitrary $a \in N$, there is a constant $\lambda$ such that $a-\lambda e \in N_{1}$. But then $a\left(M_{0}\right)-\lambda=a\left(M_{1}\right)-\lambda$ and $N$ does not separate the points of $\mathfrak{M}(B)$. This contradiction completes the proof of the theorem.

\section{BIBLIOGRAPHY}

1. H. S. Bear, Thesis, 1957, University of California at Berkeley.

2. P. Civin and B. Yood, Regular Banach algebras with a countable space of maximal regular ideals, Proc. Amer. Math. Soc. vol. 7 (1957) pp. 1005-1010.

3. H. Helson and F. Quigley, Maximal algebras of continuous functions, Proc. Amer. Math. Soc. vol. 8 (1957) pp. 111-114.

4. K. Hoffman and I. M. Singer, Maximal subalgebras of $C(\Gamma)$, Amer. J. Math. vol. 79 (1957) pp. 295-305.

5. L. H. Loomis, An introduction to abstract harmonic analysis, New York, D. Van Nostrand Co., 1953.

6. J. Wermer, Subalgebras of the algebra of all continuous complex valued functions on the circle, Amer. J. Math. vol. 78 (1956) pp. 225-242.

UNIVERSITY OF OREGON 